Observatory, and 55 in WEBCAST2). Eighty-six out 169 aneurysms were located at middle cerebral artery (50.9\%), 36/ 169 at anterior communicating artery (21.3\%), 30/169 at basilar artery $(17.8 \%)$, and $17 / 169$ at internal carotid artery terminus (10.1\%). Placement of the WEB device was feasible in $163 / 169$ aneurysms (96.4\%). Morbidity and mortality at one month were reported in $4 / 168$ patients $(2.4 \%)$ and $0 / 168$ patients $(0.0 \%)$, respectively. At 6 months, morbidity and mortality were reported in $1 / 99$ patients $(1.0 \%)$ and $3 / 99$ patients (3.0\%), respectively (there was no follow-up at 6 months in French Observatory).

Anatomical results at 6 months (WEBCAST and WEBCAST2) were complete aneurysm occlusion in 51/90 aneurysms (56.6\%), neck remnant in 23/90 aneurysms (25.5\%), and aneurysm remnant in 16/90 aneurysms (16.7\%). Anatomical results at 12 months (WEBCAST and French Observatory) were complete aneurysm occlusion in 56/100 aneurysms (56.0\%), neck remnant in 26/100 aneurysms (26.0\%), and aneurysm remnant in 18/100 aneurysms (18.0\%).

Conclusion This analysis in the large cumulated population of 3 GCP studies confirms the high safety of WEB treatment with low morbidity and mortality at one month and 6 months. At 6 and 12 months, adequate occlusion (complete aneurysm occlusion and neck remnant) was obtained in a high percentage of aneurysms (82.2\% and $82.0 \%$, respectively).

Disclosures L. Pierot: 2; C; Sequent. A. Molyneux: 2; C; Sequent. J. Byrne: 2; C; Sequent.

\section{P-029 TURBULENT FLOW IN THE VENOUS OUTFLOW TRACT OF PULSATILE TINNITUS PATIENTS WITH SIGMOID SINUS DIVERTICULUM}

S Kefayati, ${ }^{1} E$ Kao, ${ }^{1} \mathrm{~J}$ Liu, ${ }^{1} \mathrm{H}$ Haraldsson, ${ }^{1} \mathrm{~F}$ Faraji, ${ }^{1} \mathrm{M}$ Ballweber, ${ }^{2} \mathrm{~K}$ Meisel, ${ }^{1} \mathrm{~V}$ Halbach, ${ }^{1} \mathrm{D}$ Saloner, ${ }^{1} \mathrm{M}$ Amans. ${ }^{1}$ Radiology, UCSF, San Francisco, CA; ${ }^{2}$ Neurology, UCSF, San Francisco, $C A$

\subsection{6/neurintsurg-2016-012589.71}

Introduction/purpose Pulsatile tinnitus (PT) has been linked to multiple anatomical variants of the venous outflow tract including sigmoid sinus diverticulum (SSD). We hypothesize that turbulence generated by the SSD, which is normally preceded by a transverse sinus stenosis (TSS), can explain the source of sound. Turbulent flow has a known association to vasculogenic bruit. Advanced magnetic resonance velocimetry (MRV) was performed in cerebral venous sinuses and internal jugular veins (IJV) to obtain both volumetric velocity and turbulence maps.

Materials and methods A series of MRI protocols was performed at $3 \mathrm{~T}$ for patients with suspected venous PT including contrast-enhanced MRA (CE-MRA) to delineate the anatomy and identify SSD, 4 D-flow MR to acquire timeresolved phase (velocity) and flow-encoded magnitudes in all three directions, as well as $2 \mathrm{D}$ flow to obtain flow waveforms. Flow turbulence was quantified based on turbulence kinetic energy (TKE). Five SSD patients were evaluated, four of which had an associated TSS upstream from the SSD.

Results The TSS resulted in a jet of flow, the direction of which seemed to determine the position of the SSD downstream. The streamline visualization of the flow patterns revealed recirculation in the diverticulum. A helical flow pattern was also found adjacent to the jet flow opposite and downstream of the SSD (Figure 1). TKE mapping revealed a highly localized region of elevated TKE downstream from the stenosis extending to the opening of the diverticulum with a maximum value of $80 \mathrm{~J} / \mathrm{m}^{3}$ (Figure 2).

Discussion and Conclusion The unique flow patterns and the presence of turbulence introduced by the TSS and SSD may help explain PT in these patients. Treatment of TSS with PTA and stent implantation, or treatment of the SSD with coil embolization can potentially alter the TKE level and pattern.

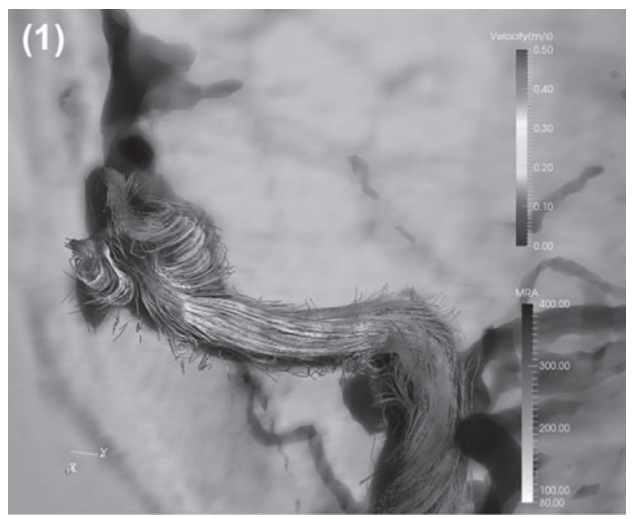

Abstract P-029 Figure 1 A tilted anterior-posterior projection of the venous sinuses and IJV in a PT patient with the SSD and upstream TSS. MRV-based streamlines colored by the magnitude of the velocity are overlaid on to the geometry captured by CE-MRA (black)

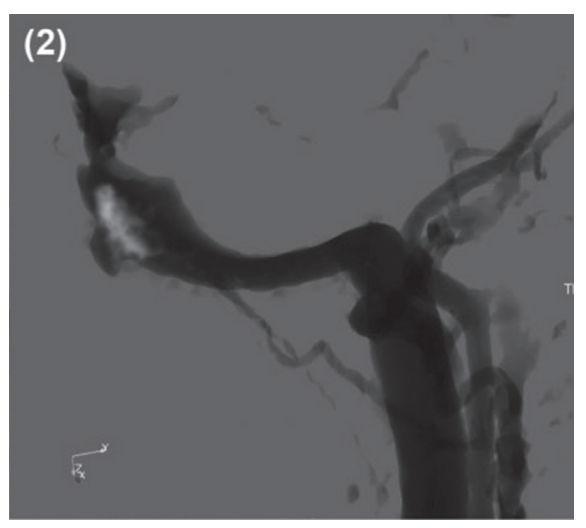

Abstract P-029 Figure 2 MRV-based measurement of the TKE in the same PT patient as shown in Figure 1. The elevated TKE region is highly localized to the downstream of the stenosis and extends into the opening of the diverticulum

Disclosures S. Kefayati: None. E. Kao: None. J. Liu: None. H. Haraldsson: None. F. Faraji: None. M. Ballweber: None. K. Meisel: None. V. Halbach: None. D. Saloner: None. M. Amans: None.

\section{P-030 PRELIMINARY EXPERIENCE WITH THE NEUROFORM ATLAS STENT IN STENT-ASSISTED ANEURYSM COILING}

G Ciccio, MD, S Smajda, MD, T Robert, MD, H Redjem, MD, R Blanc, MD, C Ruiz Guerrero, J Desilles, H Takezawa, P Sasannejad, M Piotin. Interventional Neuroradiology, Fondation Rothschild Hospital, Paris, France

10.1136/neurintsurg-2016-012589.72 